\title{
Adaptación y evaluación psicométrica de la escala de Identificación Organizacional con Centros Educativos en estudiantado de ingeniería chileno
}

\section{Adaptation and psychometric evaluation of the Organizational Identification scale with Educational Centers in Chilean engineering students}

Recibido: 17 de noviembre de 2021 | Aprobado: 1 de diciembre de 2021

Ana B. Bernardo** Universidad de Oviedo, España bernardoana@uniovi.es

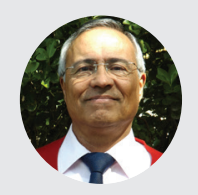

Alejandro Díaz-Mujica*** Universidad de Concepción, Chile adiazm@udec.cl

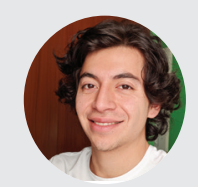

Diego Chacano Osses ${ }^{* \star * *}$ Universidad de

Concepción, Chile chacanistico@gmail.com

\section{Resumen}

La identificación con la carrera es un factor importante para el compromiso, desempeño y el abandono de los estudios. En la educación en ingeniería se vuelve clave debido al déficit en estas variables. La necesidad de un instrumento actualizado y preciso para su medición motivó la posibilidad de adaptación de la escala de Identificación Organizacional con Centros Educativos (IOCE) para esta población. Así, el objetivo de la presente investigación es mostrar el proceso de adaptación y evaluación llevada a cabo. Para ello, se realizó una investigación instrumental y transversal cuyos participantes fueron 287 estudiantes de ingeniería en una universidad chilena, que testeó 3 modelos potenciales. El mejor ajuste se obtuvo con un modelo que considera 2 factores de primer orden y uno de segundo orden. Además, las medidas de confiabilidad y validez de criterio fueron favorables. Se concluye la utilidad del IOCE para la evaluación del constructo en el estudiantado de ingeniería, teniendo en cuenta adaptaciones en su forma de pesquisa y de la comprensión sobre sus factores.

Palabras clave: Identificación, pertenencia, educación en ingeniería, compromiso académico, abandono.

\footnotetext{
* Magíster en Política y Gobierno. Profesor asistente, San Sebastián, sede Concepción, Chile. Para contactar al autor: jorge.maluenda@uss.cl

** Doctora en Psicología. Profesora titular, Universidad de Oviedo, España. Para contactar a la autora: bernardoana@uniovi.es

*** Doctor en Psicología. Profesor titular, Universidad de Concepción, Chile. Para contactar al autor: adiazm@udec.cl

**** Licenciado en Psicología. Universidad de Concepción. Para contactar al autor: chacanistico@gmail.com
} 


\section{Abstract}

Study program identification is an important factor for the Study Engagement, performance, and abandonment of studies. In engineering education, it becomes key due to the deficit in these variables. The need for an updated and precise instrument for its measurement motivated the present investigation to adapt and evaluate the Organizational Identification with Educational Centers (IOCE) scale for this population. An instrumental and cross-sectional research was carried out whose participants were 287 engineering students at a Chilean university that tested 3 potential models. The best fit was obtained with a model that considers 2 factors of the first order and one of the second order. Furthermore, the measures of reliability and criterion validity were favorable. The usefulness of the IOCE for the evaluation of the construct in engineering students is concluded, considering adaptations in their form of research and the understanding of its factors.

Keywords: Identification, belongingness, engineering education, study engagement, dropout.

\section{Introducción y marco referencial}

\subsection{Identidad organizacional en el contexto educativo \\ El vínculo entre la identidad del estudiantado y la institución en que cursa sus estudios es un aspecto relevante que considerar para mejorar la experiencia universitaria.}

Se ha propuesto que la correspondencia que el estudiantado percibe entre las características académicas de la institución en la que se integran y las características socioeconómicas y culturales del estudiantado que la conforman permiten la adecuada integración socio-académica (Leyton et al., 2012). Por el contrario, el paulatino desapego de los estudiantes frente a su carrera y sus grupos de referencia es un componente importante en el fenómeno del abandono de los estudios (Tinto, 1989).

En cuanto a la teoría de la identidad social, Tajfel (1974) propone que esta se define como la identidad social es el vínculo psicológico que permite la unión de la persona con su grupo de referencia. El núcleo de esta teoría se encuentra en que parte importante del autoconcepto de un individuo se produce por la combinación de su identidad social (saber que pertenece a un grupo), la significación emocional y el valor que tiene para él dicha pertenencia (Scandroglio et al., 2008). La identidad social ocurre cuando el individuo se categoriza positiva o negativamente por pertenecer a un grupo y genera un afecto por el efecto de ser consciente de su pertenencia al grupo (Mercado y Hernández, 2010).
Un concepto que ha adquirido valor por su pragmatismo y aplicabilidad es el de identidad organizacional. Su conceptualización fue planteada por primera vez por Ashforth y Mael basándose en los postulados de la teoría de la identidad social (Ashforth y Mael, 1989). La identidad organizacional involucra la percepción de un vínculo o lazo psicológico de un individuo con la organización, lo que implica la percepción de tener similares creencias, propósitos, objetivos y/o ideas que la organización (Álvarez et al., 2014; Rocha y Böhrt, 2003). Otros autores han propuesto que la identidad organizacional incluiría el grado en que las personas unen su sentido del yo con el de un grupo, piensan de sí mismos y del grupo en términos similares y se definen a sí mismos en términos de su membresía (Tyler y Blader, 2000).

En resumen, el estudiantado se siente perteneciente a una organización cuando comparte su cultura y valores, siente deseos de permanecer, se percibe aceptado, valorado y forma parte en las actividades de grupo. De ahí que, la máxima pertenencia ocurre cuando el individuo expresa su voluntad para participar conscientemente de un grupo u organización (Capello, 2015). Esto implica que, las personas que integran su self con el sentido colectivo, manifiestan un cambio en su identidad $\mathrm{y}$, como consecuencia, cambian la forma en como experimentan y se relacionan con una organización (Tyler y Blader, 2000). De este modo, las personas pertenecientes se definen a sí mismas, en parte, por los mismos atributos que definen a la organización de referencia (Boros y Curseu, 2012). 
En la operacionalización de los conceptos existen diferencias sustantivas entre la identidad social y la identificación organizacional. En primer lugar, la identidad organizacional incluye a grupos interactivos a diferencia de la identidad social que contempla el grupo como construcción mental (Henry et al., 1999). En segundo lugar, la identidad organizacional alude a procesos intragrupales mientras que, la social, a procesos intra e intergrupales. Por último, la identidad social es entendida como variable dicotómica (pertenecer o no a un grupo) (Turner, 1975), mientras que, la identificación organizacional se mide como una variable continua (Hinkle, et al., 1989).

En el contexto educativo, la identificación organizacional, o identificación con la organización educativa, ocurriría cuando el estudiantado percibe una alta alineación entre sus valores y los valores de la organización (Ashforth y Mael, 1989; Capello, 2015). En consecuencia, el estudiantado se percibe en estrecha conexión con su institución, facultad y/o escuela (Torres y Solberg, 2001). La identificación organizacional también ha sido descrita por Leyton et al. (2012) como la sintonía del estudiantado respecto de los valores e identidad de una institución, además de sentirse ajustado al entorno social y académico.

Adicionalmente, implica sentirse un miembro importante y respetado en su propio entorno de estudios (Alkan, 2014) donde el sentido de pertenencia se produce a partir del juicio sobre el ajuste del estudiantado al nuevo escenario universitario, basándose en el sentido de afiliación e identificación con la comunidad universitaria (Hoffman et al., 2003).

Los estudiantes se sienten atraídos por las instituciones y los individuos cuando perciben que tienen características similares a las suyas con las cuales compartir sentimientos, opiniones y valores, lo que puede influir en su vinculación permanente con la institución.

Por otra parte, para el estudiantado universitario la carrera que estudian y el grupo con el que se relacionan es una de las categorías sociales más importantes, lo que contribuye a la construcción de su identidad social y, en gran medida, define su vida (Yáñez, et al., 2006). Cuando el estudiante se siente perteneciente a su carrera, siente que tiene una participación valiosa, ya que mantiene relaciones con sus pares y percibe preocupación de parte de los académicos hacia su persona (Hoffman et al., 2003).

Por el contrario, la desidentificación ha sido definida como un estado afectivo en que los estudiantes no se sienten pertenecientes a su centro de estudio ni lo valoran, tampoco se sienten aceptados por los demás miembros de la organización, lo que podría influir en su nivel de compromiso e incluso generar desconfianza hacia la institución y sus miembros.

En investigaciones previas de tipo asociativopredictivo en estudiantado universitario, se ha observado que la identificación organizacional es un predictor fuerte para el compromiso académico (Di Battista et al., 2014; Finn, 1993; Wilkins et al., 2016). Esta tiene un efecto sobre el compromiso con su institución, lo que estimula al estudiantado a participar en las actividades, a trabajar más por las metas colectivas y apoyar más a sus pares. Además, los estudiantes adquieren conductas más identitarias como vestir logos e insignias (Boros y Curseu, 2012). También se ha observado que los estudiantes que se sienten pertenecientes a una comunidad de aprendizaje manifiestan mayores niveles de apoyo percibido de los pares y profesores, de comprensión empática de sus docentes, de comodidad en el aula y mayor sentido de pertenencia a la carrera que estudian (Hoffman et al., 2003). Adicionalmente, se ha reportado que el conocimiento de la "marca universidad" y el prestigio de esta marca se relaciona con la identificación del estudiantado con la misma. A su vez, los estudiantes que manifiestan sentirse identificados, perciben su futuro entrelazado con la universidad, lo que los impulsa a realizar acciones que apoyen a la universidad (Balaji, et al., 2016).

En el estudiantado universitario de origen socioeconómico vulnerable, una investigación cualitativa mostró que estos perciben que las diferencias económicas y sociales con sus compañeros (discrepancia identitaria) se transforman en barreras para su integración, vínculo social y sentido de pertenencia al grupo (Leyton et al., 2012). 
Por otro lado, en un estudio asociativo-predictivo en estudiantado universitario se observó que el sentido de pertenencia a la carrera y el compromiso académico fueron predictores directos y negativos de la intención de abandono de los estudios (Alkan, 2014).

El vínculo que ha sido descrito entre el sentido de pertenencia y el compromiso académico adquiere relevancia adicional, debido a la relación entre este último y variables fundamentales para el proceso de enseñanza-aprendizaje como son el desempeño académico (Maluenda et al., 2021a; MaluendaAlbornoz et al., 2021) y el abandono de los estudios (Díaz-Mujica et al., 2018; Maluenda et al., 2020; Maluenda et al., 2021).

En el contexto de educación en ingeniería en Chile existe la preocupación por el bajo compromiso de los y las estudiantes con sus estudios y asociado a ello, altas cifras de abandono de los estudios, especialmente en algunas especialidades (DíazMujica et al., 2018; Esteban, et al., 2017; Pérez et al., 2017). Además, existe escasa investigación que asocie resultados vinculados al proceso de enseñanza-aprendizaje y la identificación organizacional, en estudiantado de ingeniería.

Como ya se ha mencionado, la investigación en torno a la importancia de la identificación con la organización educativa ha sido escasa en el contexto de la educación en ingeniería. Una razón para ello es la falta de instrumentos actualizados y pertinentes a las particularidades de este tipo de población. Por estas razones, el objetivo de la presente investigación consiste en mostrar el proceso de evaluación llevado a cabo a las propiedades psicométricas del IOCE para su uso con estudiantado de ingeniería.

1.2 Medición de la identidad organizacional en el contexto universitario: La escala de identificación organizacional de Cheney

La escala utilizada más ampliamente para investigar la identificación organizacional es el Organizational Questionnaire, desarrollado por Cheney (1982), constituyendo la primera operacionalización del concepto de identificación organizacional. Este se compone de tres dimensiones: a) Membresía, o el grado en que el autoconcepto de las personas está vinculado a la organización; b) Lealtad, o el apoyo y defensa que los empleados hacen de la organización; y c) Similaridad, o la percepción de metas e intereses compartidos con otros integrantes de la organización (Yáñez et al., 2006). El estudio original encontró que la escala tenía una estructura unidimensional y presentaba un alto índice de consistencia interna (Alfa de Cronbach).

Gautam et al. (2004) desarrollaron una versión abreviada de 8 ítems de la escala de identificación organizacional encontrando adecuados índices de ajuste para el modelo unidimensional $\left(X^{2}=45.23\right.$, d.f. $=20, p<0.01$; RMSEA $=0.05 ; C F I=0.97$ ) y cargas factoriales que fluctuaron entre 0.30 y 0.74 (Gautam et al., 2004).

Previamente, una investigación realizada con 724 estudiantes universitarios, Yáñez et al. (2006) adaptaron y evaluaron los índices de validez y confiabilidad de la escala abreviada por Gautam (Gautam et al., 2004) para ser utilizada en el contexto educativo chileno. El proceso de adaptación de la escala abreviada de Identificación Organizacional con Centro Educativos (IOCE) se realizó a través de dos fases: a) Evaluación de las propiedades psicométricas de la escala; b) Validez convergente de la escala en contraste con la escala de confianza hacia los docentes. Se utilizó Análisis Factorial Exploratorio con extracción de componentes principales y rotación Varimax, considerando autovalores mayores que 1 para establecer el número de factores a retener. El análisis arrojó 1 factor que explicó el 41,3\% de la varianza y una confiabilidad de 0.80 a través del Alfa de Cronbach, y se encontró una correlación positiva y significativa entre el IOCE y la Escala de Confianza hacia los Docentes. El cuestionario quedó conformado por 8 ítems que se responden en una escala Likert de 1 a 7 donde 1 equivale al máximo desacuerdo y 7 equivale al máximo acuerdo (Tabla 1). 
Tabla 1.

Propiedades psicométricas por ítem del IOCE

\begin{tabular}{|c|c|c|}
\hline Ítem & $\begin{array}{l}\text { Correlación } \\
\text { inter-escala }\end{array}$ & $\begin{array}{l}\text { Carga } \\
\text { factorial }\end{array}$ \\
\hline $\begin{array}{l}\text { 1. Son muy similares mis valores y los valores que } \\
\text { predominan al interior de mi carrera. }\end{array}$ & 0.55 & 0.69 \\
\hline $\begin{array}{l}\text { 2. Me describiría a mí mismo ante los otros diciéndoles: } \\
\text { "Realmente me siento formando parte de la carrera que } \\
\text { estoy estudiando". }\end{array}$ & 0.61 & 0.75 \\
\hline $\begin{array}{l}\text { 3. Describiría mi lugar de estudio como una gran familia } \\
\text { en la que mayoría de los estudiantes tienen un } \\
\text { sentimiento de pertenencia con la carrera. }\end{array}$ & 0.51 & 0.66 \\
\hline $\begin{array}{l}\text { 4. Siento que tengo mucho en común con los } \\
\text { estudiantes de mi carrera. }\end{array}$ & 0.51 & 0.65 \\
\hline $\begin{array}{l}\text { 5. Siento que tengo poco en común con los docentes } \\
\text { de mi carrera. }\end{array}$ & 0.44 & 0.57 \\
\hline $\begin{array}{l}\text { 6. Encuentro difícil estar de acuerdo con quienes } \\
\text { dirigen la carrera sobre los criterios que utilizan para } \\
\text { nuestra formación profesional. }\end{array}$ & 0.43 & 0.56 \\
\hline $\begin{array}{l}\text { 7. Encuentro difícil identificarme con el lugar donde } \\
\text { estoy estudiando. }\end{array}$ & 0.53 & 0.67 \\
\hline $\begin{array}{l}\text { 8. No recomendaría a un amigo estudiar donde yo } \\
\text { estudio. }\end{array}$ & 0.44 & 0.57 \\
\hline
\end{tabular}

\section{Método}

\subsection{Participantes}

La muestra del estudio estuvo compuesta de 287 estudiantes de ingeniería, 58,82\% hombres y $41,17 \%$ mujeres pertenecientes a las 14 áreas de especialidad y a diferentes años de la carrera de ingeniería de una universidad perteneciente al Concejo de Rectores de Chile. La media de edad de los participantes fue 21,57 años y su desviación típica 1.98 años. La muestra equivale al 25,52\% del total de la población de estudio.

\subsection{Diseño y procedimiento}

El estudio se realizó con un diseño de tipo instrumental, realizado en un corte transversal de tiempo (Ato et al., 2013). El muestreo fue de tipo accidental considerando que se realizó el levantamiento de datos con el estudiantado disponible el día de la evaluación en las salas de clases.
Se realizó una revisión de contenido y lingüística del IOCE en función de su adecuación para el contexto de educación en ingeniería. Para ello, se realizaron entrevistas cognitivas con 5 estudiantes de ingeniería que consistieron en: a) contestar el instrumento, b) evaluar aquellos ítems que representaran alguna complejidad de comprensión, c) proponer mejoras específicas en su redacción. Luego, se solicitó a profesionales especialistas en educación en ingeniería (2 psicólogos y 2 académicos ingenieros) revisar la orientación y adecuación de los ítems para este tipo de estudiantado. A partir de estas dos etapas, se sugirieron algunas modificaciones (Tabla 2). Además, se les solicitó evaluar criterios éticos: a) Existencia de elementos ofensivos (raza, sexo, nivel económico, etc.) y b) Presencia de estereotipos. En este aspecto, los jueces fueron unánimes en señalar la no existencia de estos problemas. También se utilizó un criterio de contenido basado en a) ítems muy generales, b) redundancia entre ítems, c) afirmaciones poco claras, d) dimensiones no 
relevantes al constructo, e) ítems poco pertinentes al constructo, f) los ítems no cubren todos los aspectos relevantes del constructo y g) existencia de sesgo de deseabilidad social. Al respecto, los jueces estuvieron de acuerdo en la existencia de carencias vinculadas a los ítems d y e, razón por la que se emprendieron modificaciones que se detallan en la sección de resultados. Los demás aspectos fueron considerados adecuados de manera unánime.

Posteriormente, se evaluó la validez de constructo del IOCE a partir de Análisis Factorial Confirmatorio (AFC) con método de estimación de factores ML. El procedimiento consistió en analizar la matriz de correlaciones y las cargas factoriales de los ítems, cuyo criterio de calidad es que sean significativas iguales o mayores a 0.40 (Hu et al., 1999). Además, se evaluaron los índices de ajuste a partir de valores de Chi-cuadrado no significativo $\mathrm{p} \geq 0.05$ ( $\mathrm{Hu}$ et al., 1999), Error de Aproximación Cuadrático Medio (RMSEA) menor de 0.08, Índice de Ajuste Comparativo (CFI) e Índice Tucker-Lewis (TLI) mayores de 0.90 (Hair et al., 2014).

La evaluación de la validez de criterio se realizó contrastando el puntaje global de la escala respecto de la intención de abandono y de permanencia en la carrera reportada donde una correlación baja equivale a un $r<0.03$, una correlación moderada fluctúa entre 0.3 y 0.5 y una correlación alta equivale a $r>0.05$.

Por último, se evaluó la confiabilidad del instrumento en relación a la escala global a través del índice Alfa de Cronbach y el índice Omega de McDonald's (Hu et al., 1999). Se utilizó este segundo indicador debido a que, tal como se ha indicado en la literatura, se prefiere el índice Omega debido a que su calidad no se ve afectada por la cantidad de ítems del cuestionario y el volumen de la muestra, pudiendo ser utilizado sin problemas con variables no continuas a diferencia del coeficiente Alfa (Ventura-León y Caycho-Rodríguez, 2017).

\subsection{Recolección de datos}

Los participantes fueron seleccionados mediante la gestión de los jefes de carrera. Estos facilitaron los permisos y gestionaron las aplicaciones en las mismas salas de clase donde tienen actividades regularmente. Las evaluaciones se realizaron antes del inicio de clases, a través de cuestionarios en papel previa firma del consentimiento informado que incorporó todos los aspectos éticos necesarios para la investigación en ciencias humanas. No se dio ningún incentivo por participar. La información fue recolectada durante los meses de agosto y septiembre del año 2019 (segundo semestre académico en Chile).

\section{Resultados}

\subsection{Adaptación de ítems}

La entrevista cognitiva con las y los estudiantes y la evaluación de los jueces trajo como resultado una serie de recomendaciones de mejora para el instrumento. Estas recomendaciones fueron evaluadas en función de la coherencia teórica de los ítems a modificar y en cuanto a los resultados psicométricos obtenidos previamente (Yáñez et al., 2006). La comparativa entre los ítems originales y los ítems que permanecen se puede observar en la Tabla 2.

Al respecto, se optó por reemplazar en el reactivo 6 la palabra "lugar" por la palabra "carrera". Esto porque "lugar", fue entendido por el etudiantado con relación al espacio físico más que en función del espacio social y psicológico que involucra la carrera. Además, se decidió eliminar los ítems 6 y 8 del instrumento original. En el primer caso, debido a que, a juicio de los evaluadores, el ítem reflejaría mejor la percepción de consenso respecto de la capacidad para la toma de decisiones en la carrera que un sentido de identificación con los valores y creencias de la carrera. En el segundo caso, se argumentó que la recomendación de estudio a un tercero podría estar vinculada con una serie de factores distintos de la identificación con la carrera como son la organización, la infraestructura y tecnología, el costo del arancel, entre otros. Estos dos ítems coinciden, además, con aquellos que obtuvieron más baja carga factorial y correlación inter-escala en el estudio original (Yáñez et al., 2006).

Por otra parte, se sugirió incorporar un ítem que se refiriera directamente al sentido de pertenencia. Por esta razón, se incorporó el ítem "Me siento perteneciente a la carrera que estoy estudiando" con la finalidad de atender a esta solicitud. 
La nueva propuesta fue analizada por el equipo de investigación con la finalidad de verificar su ajuste conceptual respecto del constructo a evaluar.

Al respecto, se propuso el análisis de 3 modelos distintos que tendrían potencial de ajuste tanto conceptual como estadístico: a) El modelo original conformado por un solo factor; b) Un modelo conformado por dos factores, Identificación con los valores de la carrera (I.V.C) y Sentido de pertenencia a la carrera (S.P.C); c) Un modelo de segundo orden donde los factores serían los mismos que en el caso $b$, pero donde además se evaluaría la existencia de un factor de orden superior (I.C).

Tabla 2. Ítems originales v/s ítems modificados

\section{Ítem modificado}

Ítem original

1. Son muy similares mis 1. Son muy similares mis valores y los valores que valores y los valores que predominan al interior de mi predominan al interior de mi carrera. carrera.

2. Siento que tengo mucho en 2. Siento que tengo mucho en común con los estudiantes de común con los estudiantes de mi carrera mi carrera.

3. Siento que tengo poco en 3. Siento que tengo poco en común con los docentes de mi común con los docentes de mi carrera. carrera.

4. Encuentro difícil identifi- 4. Encuentro difícil identificarme carme con la carrera que estoy con el lugar donde estoy estudiestudiando. ando.

5. Me describiría a mí mismo ante los otros diciéndoles: "Realmente me siento formando parte de la carrera que estoy estudiando".

6. Describiría mi lugar de estudio como una gran familia en la que mayoría de los estudiantes tienen un sentimiento de pertenencia con la carrera.

5. Me describiría a mí mismo ante los otros diciéndoles: "Realmente me siento formando parte de la carrera donde estoy estudiando".

6. Describiría mi lugar de estudio como una gran familia en la que mayoría de los estudiantes tienen un sentimiento de pertenencia con la carrera.

7. Encuentro difícil estar de 7. Me siento perteneciente a la acuerdo con quienes dirigen la carrera que estoy estudiando carrera sobre los criterios que (ítem nuevo). utilizan para nuestra formación profesional (ítem eliminado).

8. No recomendaría a un amigo estudiar donde yo estudio (ítem eliminado).

Las dos últimas propuestas distinguirían entre los ítems relacionados con el sentir un vínculo con las ideas, creencias y valores de la carrera (Álvarez et al., 2014; Rocha y Böhrt, 2003) y aquellos relacionados con percibirse miembro de la carrera (Mercado y Hernández, 2010) lo que a juicio de los autores de este artículo corresponden a subdimensiones distintas de la identificación con la organización (carrera).

\subsection{Resultados Análisis Factorial Confirmatorio}

Se testaron los 3 modelos que desde el punto de vista teórico y estadístico suponían potencial de ajuste. El primer modelo testeado consistió en considerar todos los ítems como parte de un solo factor de modo idéntico a la propuesta original (Yáñez et al., 2006). Este mostró un ajuste aceptable a partir del índice RMSEA <.08 (95\% IC: .130-.184) y valores en los índices CFI (.874) y TLI (.811) con valores fuera de los márgenes aceptados por la literatura (Hair, et al., 2014). Las cargas factoriales fluctuaron entre .426 y .872 superando la recomendación de la literatura (Figura 1). El índice $X^{2}$ y su corrección a través del ratio $X^{2 /}$ gl (8.02) también estuvieron fuera de los límites recomendados por la literatura (Hair, et al., 2014).

\section{Figura 1.}

Configuración factorial del IOCE para el modelo de 1 factor

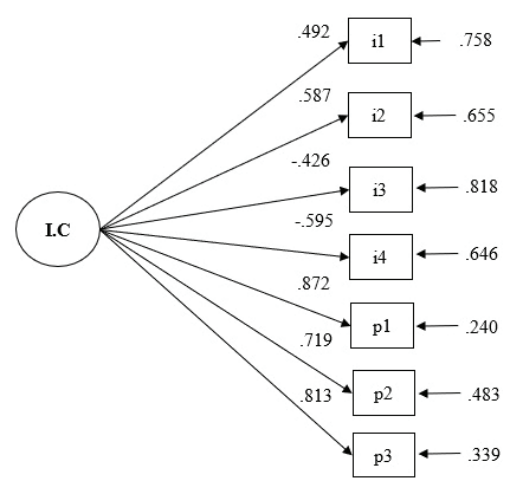

El segundo modelo, consistió en agrupar en un primer factor las variables $n^{\circ} 1$ a la $n^{\circ} 4$ debido a su vinculación teórica coherente con la idea de sentido de identificación del estudiante con la carrera, es decir, la percepción de un vínculo entre las creencias y valores del estudiantado y aquellas que la carrera representa. El segundo factor se conformó con los ítems $n^{\circ} 5$ al $n^{\circ} 7$, unidos conceptualmente por la idea de pertenencia o, en otras palabras, la percepción de membresía en la carrera (sentirse parte).

Este mostró un ajuste aceptable a partir del índice RMSEA <.08 (95\% IC: .116-.172) y del índice CFI 
(.902) (Hair, et al., 2014). Por su parte, tanto el índice TLI (.842) como $x^{2}$ y el ratio $x^{2} / g l(7.42)$ se mostraron fuera de los márgenes aceptados por la literatura (Hair, et al., 2014). Las cargas factoriales para el factor uno, fluctuaron entre .480 y .696, y para el factor 2 entre .729 y .883 (Figura 2).

\section{Figura 2.}

Configuración factorial del IOCE para el modelo de 2 factores.

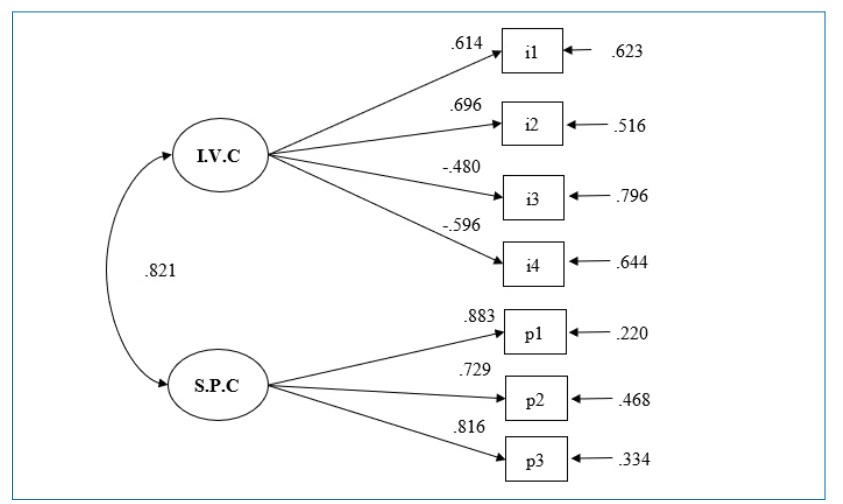

El último modelo testeado consideró como factores de primer orden la Identificación con los Valores de la Carrera (ítems del 1 al 14) y el Sentido de Pertenencia a la Carrera (ítems del 5 al 7 ) de forma idéntica al modelo anterior. Adicionalmente, se testeó la existencia de un factor de segundo orden (Identificación con la carrera). Este modelo mostró un buen ajuste en el índice RMSEA <.05 (95\% IC: .121 - .180). El ajuste en los índices CFI (.915), TLI (.901) y el ratio X²/gl (4.02) estuvo dentro del rango de valores aceptable. El único valor fuera del margen sugerido por la literatura fue $X^{2}$ (Hair, et al., 2014). Sin embargo, es relevante indicar que es $X^{2}$ tiende a desajustarse con muestras de gran tamaño. Las cargas factoriales para el factor uno, fluctuaron entre .480 y .696 para el factor 1 y para el factor 2 entre .729 y .883 (Figura 3).

\subsection{Confiabilidad}

La evaluación de la confiabilidad del instrumento se realizó a través de dos medidas: el índice Alfa de Cronbach y el índice Omega de McDonald's. Los valores obtenidos para la escala global (I.C) reflejan altos índices de confiabilidad ( $\omega=.839 ; a=.836)$. En el caso del factor I.V.C, los datos muestran un valor más bajo pero dentro del rango aceptable $(\omega=.690$; $\mathrm{a}=.678$ ). Por último, en el caso del factor S.P.C, se observa una alta confiabilidad ( $\omega=.849 ; \mathrm{a}=.852)$.

\section{Figura 3.}

Configuración factorial del IOCE para el modelo de segundo orden.

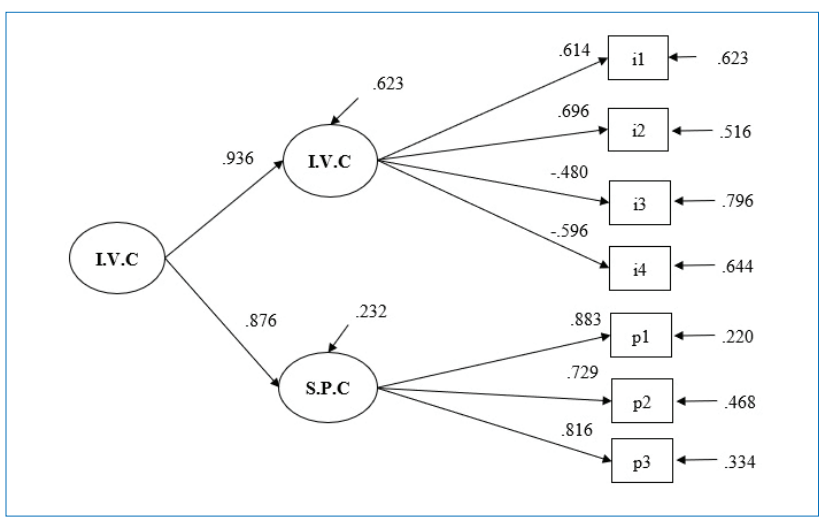

\subsection{Validez de criterio}

El análisis de la validez de criterio del instrumento mostró una correlación moderada del factor I.V.C respecto de la intención de abandono y de permanencia en la carrera. Por su parte, tanto la escala global como el factor S.P.C mostraron una correlación fuerte con ambos criterios (Tabla 3).

\section{Tabla 3.}

Correlación de la escala global y el factor S.P.C

\begin{tabular}{|c|c|c|c|c|c|c|}
\hline & & I.V.C & S.P.C & I.C & Int. Abandono & Int. Permanencia \\
\hline \multirow[t]{2}{*}{ I.V.C } & R de Pearson & - & - & - & - & \\
\hline & Valor $\mathrm{p}$ & - & - & - & - & \\
\hline \multirow[t]{2}{*}{ S.P.C } & R de Pearson & .594 & - & - & - & \\
\hline & Valor $\mathrm{p}$ & $\mathrm{P}<.001$ & - & - & - & \\
\hline \multirow[t]{2}{*}{ I.C } & R de Pearson & .889 & .897 & - & - & \\
\hline & Valor $\mathrm{p}$ & $\mathrm{P}<.001$ & $\mathrm{P}<.001$ & - & - & \\
\hline \multirow[t]{2}{*}{ Int. Abandono } & $\mathrm{R}$ de Pearson & -.306 & -.505 & -.456 & - & \\
\hline & Valor $\mathrm{p}$ & $P<.001$ & $\mathrm{P}<.001$ & $\mathrm{P}<.001$ & - & \\
\hline \multirow[t]{2}{*}{$\begin{array}{c}\text { Int. } \\
\text { Permanencia }\end{array}$} & R de Pearson & .377 & .593 & .546 & -.626 & - \\
\hline & Valor $p$ & $P<.001$ & $\mathrm{P}<.001$ & $\mathrm{P}<.001$ & - & \\
\hline
\end{tabular}




\section{Discusión y conclusiones}

El objetivo de la presente investigación fue mostrar el proceso de evaluación de las propiedades psicométricas del IOCE para su uso con estudiantado de ingeniería. Para cumplir con este se llevó a cabo, en primer lugar, la adaptación de contenido del instrumento para ingeniería y, en segundo lugar, el testeo de tres modelos que tanto en términos teóricos como estadísticos mostraban potencial de ajuste.

La adaptación de contenidos realizada llevó al retiro de 2 ítems y a la incorporación de un nuevo ítem, para luego testear los modelos potenciales. La extracción y adición de ítems tuvo sustento en el análisis de los jueces y, posteriormente, de los autores del presente artículo, quienes consideraron que los ítems 6 y 8 no se ajustaron apropiadamente al contenido propio de los constructos a evaluar (identificación y pertenencia). De este modo, el instrumento quedó conformado por ítems vinculados directamente con estos dos grandes componentes incorporados en el proceso de identificación de un individuo con su organización educativa.

El testeo de un modelo de un solo factor se sustentó en la propuesta teórica inicial de Cheney (1982) que condujo a la construcción del IOCE desarrollada por Yáñez et al., (2006). Este supone la existencia de un factor latente que explica el sentido de identificación de las y los estudiantes con la organización educativa a partir del total de los ítems considerados en el instrumento.

En la presente investigación los resultados obtenidos no permiten dar respaldo satisfactorio a este modelo, siendo el que peor ajuste muestra de los tres modelos testeados. Tal como se indicó previamente, desde el punto de vista teórico, parece existir un conjunto de ítems asociados al sentido de identificación con los valores de la carrera (I.V.C), es decir, cuán afines perciben las y los estudiantes sus propios valores con los que reconoce en su carrera. Otro conjunto de ítems apunta directamente al sentido de pertenencia a la carrera, es decir, el grado en que el estudiantado se siente parte de la carrera que cursa.

El testeo de un modelo de dos factores se sustentó en lo antes señalado asumiendo la presencia de estos dos factores (I.V.C, ítems 1 al 4; S.P.C, ítems
5 al 7). A partir del primer factor, se pretendía dar cuenta del enlace psicológico dado por la percepción que tiene un individuo de compartir las creencias, propósitos, objetivos y/o ideas de la carrera (Álvarez et al., 2014; Rocha y Böhrt, 2003). El segundo factor se encontraría relacionado con el sentido de pertenencia de las y los estudiantes a la carrera. Con este factor se intenta capturar la percepción de membresía, o el sentirse parte de la carrera (Mercado y Hernández, 2010) respecto de I.VC.

En el segundo modelo testeado tanto los índices de ajuste como las cargas factoriales mejoraron su desempeño respecto del modelo anterior. Sin embargo, los valores de TLI, $x^{2}$ y el ratio $x^{2} / g l$ mostraron valores fuera del rango sugerido por la literatura.

El tercer modelo testeado asumió los cambios anteriormente señalados, contemplando dos factores nítidamente distinguibles asociados a la identificación con los valores de la carrera (I.V.C) y el sentido de pertenencia a la carrera (S.P.C). Además, contempló el factor latente inicialmente propuesto por Yáñez et al., (2006) y presente también en la propuesta original de Cheney, pero esta vez, entendido como un factor de segundo orden que surge de los dos factores anteriormente descritos. De este modo, en términos conceptuales, la Identificación con la Carrera (I.C) se comprende como un factor latente de segundo orden compuesto por la Identificación con los Valores de la Carrera (I.V.C) y el Sentido de Pertenencia a la Carrera (S.P.C) como factores de primer orden.

En este caso, tanto los índices de ajuste como las cargas factoriales mostraron buen ajuste con excepción de $x^{2}$. Sin embargo, como ya se ha comentado, este índice suele desajustarse cuando existen muestras de gran tamaño (Hair, et al., 2014). El ratio $x^{2} / g l$, medida utilizada para complementar $X^{2}$, mostró un valor aceptable.

Este conjunto de resultados refleja un mejor ajuste que los modelos predecesores, no solo en torno a el ajuste estadísticos, sino que, también, en cuanto al modelo teórico implícito, que permitiría una explicación más clara, precisa y detallada de las mediciones realizadas con el instrumento. 
De este modo, en términos conceptuales, se entiende que la Identificación con la Carrera, es decir, el enlace psicológico que existe entre el o la estudiante y su carrera - recogido a partir del factor de segundo orden I.C - contiene dos subcomponentes: la Identificación con los Valores de la Carrera (I.V.C) dado por la percepción de coherencia entre ideas, valores y objetivos entre el o la estudiante y su carrera; y el Sentido de Pertenencia a la Carrera (S.P.C) dado por la percepción de "ser parte" o miembro valorado de la carrera que se estudia.

En cuanto al testeo de la confiabilidad del instrumento, se observó que tanto los valores obtenidos a partir del índice Alfa de Cronbach como del índice Omega de McDonald's fueron elevados, superando la recomendación entregada en la literatura que sugiere confiabilidades mayores a .60 (Hu et al., 1999). La confiabilidad por factor fue mejor en el subcomponente S.P.C. Con todo, los indicadores muestran confiabilidad en este instrumento, resultado coherente con la investigación original (Yáñez et al., 2006).

En cuanto a la validez de criterio, se observó una correlación negativa mediana entre el puntaje global de la escala y la intención de abandono de la carrera. Este resultado demuestra coherencia con la investigación previa con universitarios donde se ha observado una relación negativa mediana entre estos constructos (Alkan, 2014) y, específicamente, con estudiantado de ingeniería chileno (Maluenda, et. al, 2021a; Maluenda-Albornoz et al., 2021).

A raíz de todos los resultados presentados, es posible concluir que el instrumento muestra propiedades psicométricas ajustadas para un modelo que incluye 2 factores de primer orden y un factor de segundo orden, para su uso con estudiantado de ingeniería. Sin embargo, habrá que profundizar en la investigación al respecto que permita refinar el instrumento para mejorar los indicadores presentados, sobre todo, en cuanto al alcance de una mayor confiabilidad.

Una debilidad importante de esta investigación es la captura de una muestra que refiere a una sola institución universitaria, lo cual, plantea la necesidad de investigación futura que permita establecer conclusiones de mayor alcance en cuanto a la generalización para la población de estudiantes de ingeniería.

La contribución principal de este artículo es la obtención de las propiedades psicométricas en esta población a partir de un análisis teórico y empírico robusto. Esto permitirá no solo la investigación ulterior, sino que también la disponibilidad de una herramienta de medición del proceso de identificación de las y los estudiantes de ingeniería con su carrera que favorezca la evaluación del estado de los y las estudiantes en esta materia, además de la evaluación de procesos de intervención y mejoramiento de la experiencia universitaria. Por último, el presente artículo contribuye a la actualización de la investigación en esta materia.

\section{Agradecimientos/Reconocimientos}

Este trabajo de investigación fue realizado con aportes de la beca de doctorado nacional de CONICYT: CONICYT-PCHA/Doctorado Nacional/2018-21180223.

\section{Referencias bibliográficas}

Alkan, N. (2014). Humor, loneliness and acceptance: Predictors of university drop-out intentions. Precedia Social and Behavioral Sciences, 152, 1079-1086. https:// doi.org/10.1016/j.sbspro.2014.09.278

Álvarez, A., Ionela, A., Irene, M., Marrero, T., Mas, L. y Muñoz, M. (2014). Identificación Organizacional y Satisfacción Laboral: Diferencia entre Empresas Públicas y Privadas. REIDOCREA, 3(5), 34-40.

Ashforth, B. E. y Mael, F. (1989). Social Identity Theory and the Organization. Aca, 14(1), 2039.

Ato, M., López, J. J. y Benavente, A. (2013). Un sistema de clasificación de los diseños de investigación en psicología. Anales de Psicología, 29(3 (octubre), 1038-1059. https:// doi.org/10.6018/analesps.29.3.178511

Balaji, M. S., Kumar, S. y Sadeque, S. (2016). Antecedents and consequences of university brand identification. Journal of Bussiness Research. https://doi. org/10.1016/j.jbusres.2016.01.017 
Boros, S. y Curseu, P. L. (2012). To be or not to be .... identified. Explorations of students' (dis)identification in a Romanian university. Psihologia Resurselor Umame, 10(1), 57-69.

Capello, H. (2015). La identidad universitaria. La construcción del concepto. Revista Internacional de Ciencias Sociales y Humanidades, 25(2), 33-53.

Cheney, G. (1982). Organizational identification as process and product: A field study Unpublished master's thesis, Purdue University.

Di Battista, S., Pivetti, M. y Berti, C. (2014). Engagement in the university context: exploring the role of a sense of justice and social identification. Soc Psychol Educ, 17, 471-490. https://doi.org/10.1007/s11218$\underline{014-9255-9}$

Díaz-Mujica, A., García, D., López, Y., Maluenda, J., Hernández, H. y Pérez-Villalobos, M. (2018). Mediación del ajuste académico entre variables cognitivo-motivacionales $y$ la intención de abandono en primer año de universidad. In Octava conferencia latinoamericana sobre el abandono en la educación superior. (pp. 213-222). Panamá City.

Esteban, M., Bernardo, A., Tuero, E. y Cervero, A. (2017). Variables influyentes en progreso académico y permanencia en la universidad. European Journal of Education and Psychology, (10), 75-81.

Finn, J. (1993). School engagement \& students at risk. Washington.

Fredricks, J. y Mccolskey, W. (2012). The Measurement of Student Engagement: A Comparative Analysis of Various Methods and Studen Self-report Instruments. In C. Christenson, S, Reschly, A., Wylie (Ed.), Handbook of Research on Student Engagement. (pp. 763-782). New York: Springer-Verlag. https://doi.org/10.1007/978-1-4614-2018-7
Gautam, T., Van Dick, R. y Wagner, U. (2004). Organizational identification and organizational commitment: Distinct aspects of two related concepts. Asian Journal of Social Psychology, 7, 301-315. https://doi. org/10.1111/j.1467-839X.2004.00150.x

Hair, J., Black, W., Babin, B. y Anderson, R. (2014). Multivariate Data Analysis (Seventh Ed). Edinburg Gate: Pearson.

Henry, K., Arrow, H., y Carini, B. (1999). A tripartite model of group identification. Theory and Measurement. Small Group Research, 30(5), 558-581. https://doi.org/10. 1177\%2F104649649903000504

Hinkle, S., Taylor, L., Fox-cardamone, L. y Crook, K. (1989). Intragroup identification and intergroup differentiation: A multicomponent approach. British Journal of Social Psychology, 28, 305-317. https://doi. org/10.1111/j.2044-8309.1989.tb00874.x

Hoffman, M., Richmond, J., Morrow, J. y Salomone, K. (2003). Investigatin "sense of belonging" in first-year college students. Journal of College Student Retention, 4(3), 227-256. https://doi.org/10.2190/DRYCCXQ9-JQ8V-HT4V

Hu, L., Bentler, P. M. y Hu, L. (1999). Cutoff criteria for fit indexes in covariance structure analysis: Conventional criteria versus new alternatives. StructuralEquationModeling, 6(1),1-55.https:// doi.org/10.1080/10705519909540118

Leyton, D., Vásquez, A. y Fuenzalida, V. (2012). La experiencia de estudiantes de contextos vulnerables en diferentes instituciones de educación superior universitaria (IESU): resultados de investigación. Calidad En La Educación, (37), 61-97. http://dx.doi. org/10.4067/S0718-45652012000200003

Maluenda, J., Flores-Oyarzo, G., Bernardo, A. y Díaz-Mujica, A. (2021). Correlatos conductuales del compromiso académico en estudiantes de ingeniería chilenos. Trilogía, 13(24). https://doi. org/10.22430/21457778.1754 
Maluenda, J., Varas, M., Riffo, M. y Díaz, A. (2021a). Predictores socio-académicos del Study Engagement en estudiantes de primer año de ingeniería. Estudios pedagógicos (Valdivia), 47(1), 235-250. https://dx.doi.org/10.4067/s071807052021000100235

Maluenda-Albornoz, J., Gutiérrez, A. B., GalveGonzález, C., Flores-Oyarzo, G., InfanteVillagrán, V. y Díaz-Mujica, A. (2021). Variables predictoras de la expectativa de desempeño y la intención de abandono en contexto de educación virtual de emergencia en estudiantes universitarios chilenos. RECIE. Revista Caribeña De Investigación Educativa, 5(2), 81-91. https://doi.org/10.32541/recie.2021. v5i2.pp81-91

Maluenda, J., Varas, M., Díaz, A. y Bernardo, A. (2020). Propiedades psicométricas del University Student Engagement Inventory en estudiantes de ingeniería chilenos. Revista Iberoamericana de Diagnóstico y Evaluación - e Avaliação Psicológica RIDEP, 4(57), 77-90. https://doi.org/10.21865/RIDEP57.4.06

Maluenda, J., Moraga, F. y Díaz-Mujica, A. (2019). El rol del estudiante en el fenómeno del Compromiso Académico en Educación Superior. Wimblu, 14(1), 81-94. https://dx. doi. org/ https://doi.org/10.15517/wl.v14i1.35876

Maluenda, J. y Pérez, M. V. (2018). Comportamientos asociados al compromiso académico desde la experiencia de estudiantes de ingeniería. In Octava Conferencia Latinoamericana sobre Abandono en la Educación Superior. Panamá City.

Maroco, J., Maroco, A. L., Campos, J. A. D. B. y Fredricks, J. A. (2016). University student's engagement: development of the University Student Engagement Inventory (USEl). Psicologia: Reflexão e Crítica, 29(1), 21. https://doi.org/10.1186/s41155-016-0042-8

Mercado, A. y Hernández, A. (2010). El proceso de construcción de la identidad colectiva. Convergencia, (53), 229-251.
Pérez, M., Cobo, R., Matos, L., Hernández, H., Del Valle, M. y Díaz-Mujica, A. (2017). Variables cognitivo-motivacionales como predictoras del ajuste a la vida universitaria y la intención de abandonar los estudios en estudiantes de primer año. In VII CLABES. Séptima conferencia latinoamericana sobre el abandono en la educación superior.

Rocha, M. y Böhrt, M. (2003). Tres dimensiones del compromiso organizacional: Identificación, membresía y lealtad. Ajayu, 2(1). http://www. scielo.org.bo/pdf/rap/v2n1/v2n1a8.pdf

Scandroglio, B., López, J. y Carmen, S. J. (2008). La Teoría de la Identidad Social: una síntesis crítica de sus fundamentos, evidencias y controversias. Piscothema, 20(1), 80-89.

Tajfel, H. (1974). Social identity and intergroup behavior. Social Science Information, 13, 65-93.

Tinto, V. (1989). Definir la deserción: Una cuestión de perspectiva. Revista de Educación Superior, 71, 35-51.

Torres, J. y Solberg, S. (2001). Role of Self-Efficacy, Stress, Social Integration, and Family Support in Latino College Student Persistence and Health. Journal of Vocational Behavior, 59, 53-55. https://doi.org/10.1006/ jvbe.2000.1785

Turner, J. (1975). Social comparison and social identity: Some prospects for intergroup behavior. European Journal of Social Psychology, 5(1), 5-34. https://psycnet.apa. org/doi/10.1002/ejsp.2420050102

Tyler, T. y Blader, S. (2000). Cooperation Groups: Procedural Justice, Social Identity and Behavioral Engagement. Philadelphia: Psychology Press.

Ventura-León, J. L. y Caycho-Rodríguez, T. (2017). El coeficiente Omega: un método alternativo para la estimación de la confiabilidad. Revista Latinoamericana de Ciencias Sociales, Niñez y Juventud, 15(1), 625-627. 
Wilkins, S., Butt, M. M., Kratochvil, D. y Balakrishnan, M. (2015). Studies in Higher Education The effects of social identification and organizational identification on student commitment, achievement and satisfaction in higher education. Studies in Higher Education. https://doi.org/10.1080/03075079.2015.103 4258
Yáñez, R., Perez, M. V. y Ahumada, L. (2006). Adaptación y validación de una escala de identificación organizacional con centros de estudio. Paideia, (41), 65-76. 